\title{
Microstructure, stress and texture in sputter deposited TiN thin films: effect of substrate bias
}

\section{J. Chakraborty ${ }^{1, a^{*}}$,Tias Maity ${ }^{2, b}$, Kishor Kumar $^{3, c}$ and S. Mukherjee ${ }^{3, d}$}

\author{
${ }^{1}$ Materials Science and Technology Division, CSIR-National \\ Metallurgical Laboratory, Jamshedpur-831007, India \\ ${ }^{2}$ Department of Chemical Engineering and Material Science (CHEMS), Michigan State \\ University, East Lansing, Michigan - 48824-1226, US ${ }^{3}$ Facilitation centre for Industrial plasma \\ Technology, Institute for Plasma Research, A-10/B GIDC, Sector-25, Gandhinagar - 382 016, India \\ jay@nmlindia.org, ${ }^{b}$ tiaskumar@gmail.com ${ }^{c}$ kishorkumak.k@googlemail.com, \\ dmukherji@ipr.res.in
}

Keywords: TiN, Thin films, sputtering, X-ray diffraction, texture, stress

\begin{abstract}
:
Titanium nitride thin films deposited by reactive dc magnetron sputtering under various substrate bias voltages have been investigated by X-ray diffraction. TiN thin films exhibits lattice parameter anisotropy for all bias voltages. Preferential entrapment of argon atoms in TiN lattice has been identified as the major cause of lattice parameter anisotropy. Bombardment of argon ions during film growth has produced stacking faults on $\{111\}$ planes of TiN crystal. Stacking fault probability increases with increasing substrate bias voltages. X-ray diffraction line profile analysis indicates strain anisotropy in TiN thin films.

Diffraction stress analysis by $d-\sin ^{2} \psi$ method reveals pronounced curvature in the plot of interplanar spacing $(d)$ (or corresponding lattice parameter $(a)$ ) versus $\sin ^{2} \psi$. Direction dependent elastic grain interaction has been considered as possible source of the observed anisotropic line broadening.
\end{abstract}

\section{Introduction}

Titanium nitride (TiN) thin films deposited by reactive magnetron sputtering is widely used for improving the hardness and wear resistance of materials surfaces [1]. Such functional properties critically depend on the microstructure of TiN thin films. It is well known that ion bombardment during thin film deposition can change the microstructure of such hard coating [2-4]. During the films deposition, the substrate is negatively biased (with respect to deposition chamber) so that the positively charged ions of the sputtering gas such as argon can be accelerated towards the substrate during the film growth. Negative bias voltage of the substrate can be varied in order to change the energy of the bombarding ions which is the key to change the film microstructure. In the present work, microstructure of TiN thin films deposited at various substrate bias voltages has been studied by X-ray diffraction. Intrinsic/growth stresses and crystallographic textures in TiN films have been studied as a function of substrate bias voltages (i.e. energies of the bombarding ions).

\section{Specimen preparation and characterization}

Polycrystalline titanium nitride thin films (thickness $\sim 2 \mu \mathrm{m}$ for all films) were deposited on silicon ( $\{100\} \mathrm{Si}$ ) substrate by planer reactive dc magnetron (magnetron power $\sim 270 \mathrm{~W}$ ) sputtering under various substrate bias voltages $(0 \mathrm{kV}$ to $-5 \mathrm{kV})$ using a high voltage pulsed DC power supply. The sputter target was $99.99 \%$ pure titanium and argon gas of high purity (99.99\%) was used as the sputtering gas for the magnetron targets. For the reactive deposition of titanium nitride, the reactive gas nitrogen was introduced. Operating pressure of the sputtering chamber was $3 \times 10^{-3} \mathrm{mbar}$. The magnetron was biased at -450 Volts. All depositions were done at room temperature.

As-deposited films were characterized by x-ray diffraction (XRD) using $\mathrm{Cu} K \alpha$ radiation in a PANAlytical (formerly Philips) X'pert MRD diffractometer equipped with parallel beam optics and an Eulerian cradle for the measurements of phase, stress and crystallographic texture. Phase analysis of as-deposited films was performed by x-ray diffraction measurements in Bragg-Brentano geometry (with $1^{\circ} \omega$ offset where $\omega$ is the angle of incidence of $x$-ray) over the angular range of 
$30^{\circ}$ to $90^{\circ}(2 \theta)$ with $0.04^{\circ}$ step width and 10 sec. as time/step. Diffraction patterns have been fitted with Pearson-VII functions using a peak fitting program (PROFIT, PANAlytical) to determine the peak parameters like peak maximum position, FWHM (full width at half maxima), integrated intensity etc. corresponding to $K \alpha_{1}$ radiation. Diffraction line profiles analysis has been performed by traditional Williamson-Hall method (for details see section 3.2) in order to determine the size of coherently diffracting domains (crystallite size) and the types of defects generated in TiN thin films due to ion bombardment during thin film deposition. Analysis of crystallographic texture of TiN thin films has been performed by pole figure measurements $(I(\varphi \psi))$, of 111 and 200 reflections of face centered cubic (f.c.c) TiN phase where $I, \varphi$ and $\psi$ were the peak maximum intensity, specimen rotation angle and specimen tilt angle respectively. Diffraction stress analysis has been performed by $d_{\psi}^{h l}-\sin ^{2} \psi$ method [5]. Macroscopic strain measurement of polycrystalline TiN thin films has been performed by measuring orientation dependent inter-planer lattice spacing $\left(d_{\psi}^{h k l}\right)$ corresponding to 111 reflection at various specimen tilt angles $(\psi)$.

\section{Results}

\subsection{Stacking faults and lattice parameter anisotropy in TiN films: XRD analysis}

Fig. 1(a) shows the XRD patterns corresponding to polycrystalline titanium nitride thin films deposited under various substrate bias voltages. The prominent

Bragg peaks in all XRD patterns could be indexed with respect to the f.c.c TiN structure and the corresponding Miller indices $(h k l)$ are indicated in the XRD patterns. For certain bias voltages XRD patterns show formation of small amount of $\mathrm{Ti}_{2} \mathrm{~N}$ phase (marked by squares in Fig. 1(a)). Fig. 1(b) shows so called Cohen-Wagner plot $[6,7]$ for all TiN films deposited under different bias voltages. There is a large scatter in the lattice parameter $\left(a_{h k l}\right)$ values calculated from the peak positions of different $h k l$ reflections for all bias voltages. Such scatter of the values of $a_{h k l}$ in the Cohen-Wagner plot [6,7] can be due to the presence of stacking faults (SF) in fcc TiN crystal generated due to bombardment of argon ions during the growth of TiN films. The net stacking fault probabilities $(\alpha)$ (difference between intrinsic and extrinsic fault probabilities) in $\mathrm{TiN}$ thin films have been determined from the change of the

diffraction angular separation of 111 and 200 reflections of TiN films with

respect to bulk TiN containing no stacking faults (lattice parameter: 4.2386 [8]) (as standard) (see Refs. [6,7] for the detailed method of SF probability

determination). The results of this analysis have been presented in Table-1
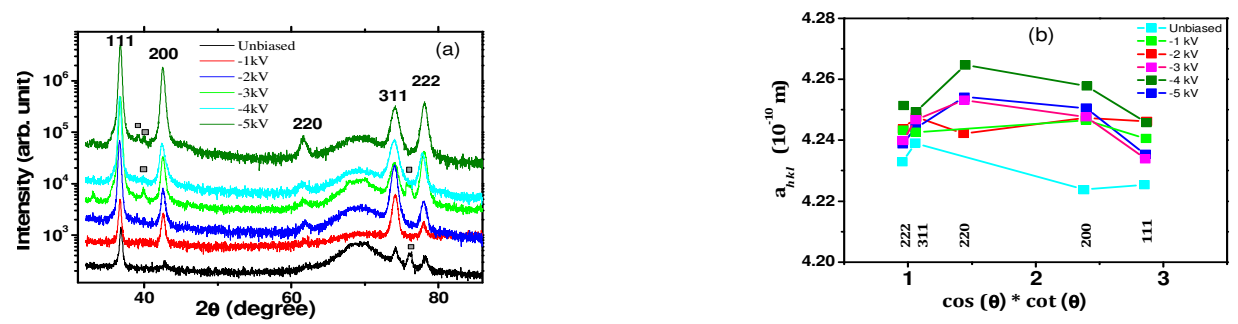

Fig.1: (a) X-ray diffraction patterns of TiN thin films; (b) Cohen-Wagner plot for lattice parameters of TiN films deposited at various substrate bias voltages.

Table-1: SF probability in TiN films deposited under various bias voltages

\begin{tabular}{|l|c|l|l|l|l|l|}
\hline Bias Voltage & $0 \mathrm{kV}$ & $-1 \mathrm{kV}$ & $-2 \mathrm{kV}$ & $-3 \mathrm{kV}$ & $-4 \mathrm{kV}$ & $-5 \mathrm{kV}$ \\
\hline $\begin{array}{l}\text { (Diffraction angular } \\
\text { separation of 111 and } \\
200) \delta \Delta(2 \theta) \text {, degree }\end{array}$ & +0.04 & -0.066 & -0.023 & -0.137 & -0.136 & -0.154 \\
\hline $\begin{array}{l}\text { Stacking fault } \\
\text { probability }(\alpha)\end{array}$ & Nil & $1.5 \times 10^{-2}$ & $0.5 \times 10^{-2}$ & $3.12 \times 10^{-2}$ & $3.12 \times 10^{-2}$ & $3.5 \times 10^{-2}$ \\
\hline
\end{tabular}

Which shows that stacking fault probability in TiN films increases with increasing substrate bias voltage (except for $-2 \mathrm{kV}$ bias voltage). Furthermore, Fig. 1(b) shows anisotropy in the lattice 
parameters $\left(a_{111}<a_{200}\right)$ for all bias voltages and the difference in the lattice parameters $\left(a_{200}-a_{111}\right)$ also increases with increasing substrate bias voltage (see sec. 4 for detailed discussion). Beyond $2 \mathrm{kV}$ substrate bias voltage, the lattice parameters show the trend $a_{111}<a_{200}<a_{220}$.

$3.2 \mathrm{X}$-ray diffraction line broadening analysis

Figs. 2(a-d) show conventional Williamson-Hall [9] plots (plot of FWHM $(\Delta K)$ as a function of the length of the diffraction vector $(K)$ ) for TiN films deposited under various substrate bias voltages ($2 \mathrm{kV},-3 \mathrm{kV},-4 \mathrm{kV}$ and $-5 \mathrm{kV})$. According to Williamson and Hall, FWHM of a diffraction line profile can be written as follows (equation (1)) where $D$ is the crystallite size, $\mathcal{E}$ is the microstrain and $K=\frac{2 \sin \theta}{\lambda}$ (i.e. $\Delta K=2 \cos \theta(\Delta \theta) / \lambda$ ) where $\theta$ and $\lambda$ are the diffraction angle and $\mathrm{x}$-ray wavelength respectively. $\Delta K=\frac{0.9}{D}+K \varepsilon \ldots \ldots \ldots \ldots \ldots \ldots \ldots \ldots . . .(1)$. If a straight line is fitted (shown by dashed lines in Fig. 2(a)(d)) to the W-H plot, then slope of the line determines the microstrain and its intercept determines the crystallite size. W-H Plot at $-2 \mathrm{kV}$ (Fig. 2(a)) shows that FWHM monotonically increases with $K$ and a straight line has been fitted (dashed line in Fig. 2(a)) whose slope and intercept determine microstrain and crystallite size respectively which are presented in Table-2 (and Table-3). However, beyond $-2 \mathrm{kV}$, all W-H plots show anisotropic dependence of FWHM on $K$ (i.e FWHM of a peak at larger $K$ value is lower than that of a peak at smaller $K$ value)) which can be observed from the FWHM values of 311 and 222 reflections in Figs. 2(b)-(d). In this case, attempt has been made to determine the crystallite size and microstrain by least square fitting of straight lines using 111/222 pair of reflections and 200/220/311 reflections separately and the results are presented in Table-2 and Table-3 respectively. For $-2 \mathrm{kV}$ substrate biasing, all reflections are considered during
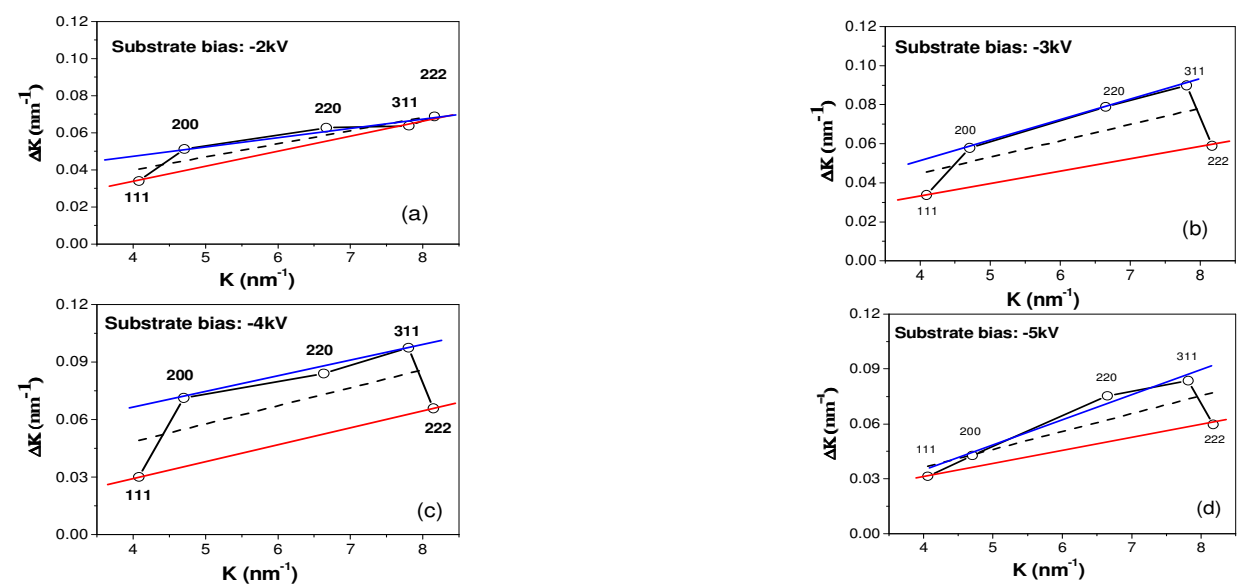

Fig.2: Williamson-Hall plots: Substrate bias voltages: (a) $-2 \mathrm{kV}$; (b) $-3 \mathrm{kV}$; (c) $-4 \mathrm{kV}$; (d) $-5 \mathrm{kV}$. Straight line fitted by least square method considering all reflections is indicated by dashed lines. 111 and 222 reflections are joined by red lines (schematic). Blue lines are schematic fitting (guide to the eye) for 200,220 and 311 reflections.

Table-2: Crystallite size and mcrostrain in $<111>$ direction (except-2kV sample)

\begin{tabular}{|l|l|l|l|l|l|l|}
\hline Bias Voltage & $0 \mathrm{kV}$ & $-1 \mathrm{kV}$ & $-2 \mathrm{kV}$ & $-3 \mathrm{kV}$ & $-4 \mathrm{kV}$ & $-5 \mathrm{kV}$ \\
\hline Crystallite size & $108 \mathrm{~nm}$ & $29 \mathrm{~nm}$ & $79 \mathrm{~nm}$ & $146 \mathrm{~nm}$ & $*$ & $131 \mathrm{~nm}$ \\
\hline Microstrain & $5.3 \times 10^{-3}$ & $7.1 \times 10^{-4}$ & $7.1 \times 10^{-3}$ & $8.5 \times 10^{-3}$ & $8.8 \times 10^{-3}$ & $3.5 \times 10^{-3}$ \\
\hline
\end{tabular}

Table-3 Crystallite size and mcrostrain by fitting 200,220 and 311 reflections

\begin{tabular}{|l|c|c|c|c|c|c|}
\hline Bias Voltage & $0 \mathrm{kV}$ & $-1 \mathrm{kV}$ & $-2 \mathrm{kV}$ & $-3 \mathrm{kV}$ & $-4 \mathrm{kV}$ & $-5 \mathrm{kV}$ \\
\hline Crystallite size & $*$ & $*$ & $79 \mathrm{~nm}$ & $21 \mathrm{~nm}$ & $28 \mathrm{~nm}$ & $*$ \\
\hline Microstrain & $*$ & $*$ & $7.1 \times 10^{-3}$ & $1.6 \times 10^{-2}$ & $8.2 \times 10^{-3}$ & $1.3 \times 10^{-2}$ \\
\hline
\end{tabular}

*Negative intercept or (and) negative slope in W-H plot; 
straight line fitting. Table- 2 shows smallest values for both microstrain and average crystallite size along $<111>$ direction of TiN films deposited under $-1 \mathrm{kV}$ substrate bias voltage. Only little increase in microstrain occurs upon increasing substrate bias voltage from $-2 \mathrm{kV}$ to $-4 \mathrm{kV}$ and at $-5 \mathrm{kV}$ bias voltage microstrain decreases (Table-2). For TiN thin films deposited under $-3 \mathrm{kV}$ and $-5 \mathrm{kV}$ bias voltages, Table- 3 shows one order higher magnitude of microstrain compared to those presented in Table-2 (i.e. microstrains along $<111>$ direction). Additionally, for TiN films deposited at $-3 \mathrm{kV}$ bias voltage, large difference in the crystallite sizes between Table- 2 and Table- 3 may be due to the presence of stacking faults on $\{111\}$ plane of TiN crystals. This can be understood from the fact that the broadening due to crystallite size involves both the effect of true crystallite size and a contribution from the stacking faults (which also acts like size effect). Therefore, due to the presence of stacking fault on $\{111\}$ planes of TiN crystal, the average size of TiN crystallites along $<111>$ direction (presented in Table-2) should differ than those obtained along other crystallographic directions. Observed anisotropy in line broadening has been discussed in section 4 . 3.4. Orientation dependent lattice parameters and Stresses in TiN films

Figs. 3(a) \& 3(b) show $a_{\psi l}^{h k l}-\operatorname{Sin}^{2} \psi$ plots corresponding to 111 reflections of TiN thin films deposited under substrate bias voltages $-2 \mathrm{kV}$ and $-4 \mathrm{kV}$ respectively.

TiN films deposited under $-4 \mathrm{kV}$ substrate bias voltage, shows pronounced curvature (Fig.3(b)) whereas little curvature is present in case of $-2 \mathrm{kV}$ biasing. $a_{\psi l}^{h k l}-\operatorname{Sin}^{2} \psi$ plots for TiN films deposited under $-3 \mathrm{kV}$ and $-5 \mathrm{kV}$ biasing also show similar curvatures. In general such curvatures or non-linearity in $a_{\psi}^{h k l}\left(\right.$ or $\left.d_{\psi}^{h k l}\right)-\operatorname{Sin}^{2} \psi$ plots can be due to (i) strain gradient across the TiN film thickness; (ii) crystallographic texture in TiN films; (biaxial alignment of TiN crystallites with $\{111\}$ out of plane texture with in-plane $\{002\}$ orientation is shown in Fig.3(c) \& (d)); (iii) direction dependent elastic grain interaction due to elastic anisotropy of TiN [10]. Straight lines are fitted in the corresponding $d_{\psi}^{h k l}-\operatorname{Sin}^{2} \psi$ plots and from the positive slopes of the lines, tensile stresses of $4 \mathrm{GPa}$ and $1.5 \mathrm{GPa}$ are obtained for $-2 \mathrm{kV}$ and $-4 \mathrm{kV}$ bias voltages respectively assuming rotationally symmetric biaxial state of stress in TiN. Obviously, large errors are involved in the stress value for $-4 \mathrm{kV}$ biasing due to poor data fitting.

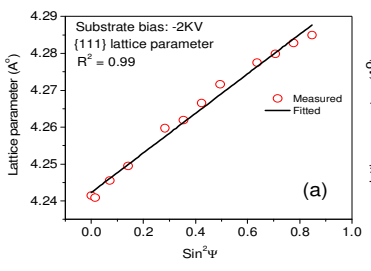

(a)

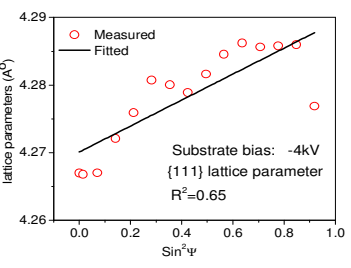

(b)

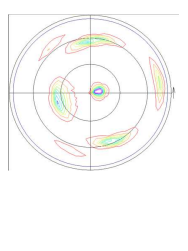

(c)

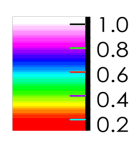

(d)

Fig.3: $a-\sin ^{2} \psi$ plots for 111 reflection of TiN films (a) $-2 \mathrm{kV}$ bias; (b) $-4 \mathrm{kV}$ bias; $\{111\}$ Pole figures of TiN thin films: bias voltages: (c) $-2 \mathrm{kV}$; (d) $-4 \mathrm{kV}$.

\section{Discussion}

Bombardment of film surface by positively charged ions (argon, nitrogen etc.) due to negative electrical biasing of the substrate may lead to the following changes in film microstructure during thin film deposition (i) lattice expansion due to preferential entrapment (i.e. depends on crystallographic orientation) of foreign atoms (argon, nitrogen etc.) in the TiN lattice; (ii) generation of lattice defects (i.e. point defects, dislocation, planar faults etc.) due to ion bombardment; (iii) ion bombardment induced crystallographic texture during film growth. In the following, obtained results in the present work are discussed with respect to the above points. For example, observed lattice parameter anisotropy in the TiN lattice $\left(a_{111}<a_{200}<a_{220}\right)$ could be due to lowest argon atoms entrapment in the (111) plane and highest in the (220) plane. Such preferential entrapment of argon ions has been observed earlier by Hultman et.al [11]. It is to note that [111] direction corresponds to the densest array of atoms in TiN crystal. Increasing bias voltage leads to 
higher energy of argon ions and more argon atoms are entrapped in (200) plane than the (111) plane which may be the reason for observed increase in the lattice parameter difference $a_{200}-a_{111}$ (see sec. 3.1) with bias voltage increase. Also, it may be noted for TiN lattice [001] is the most open channeling direction [12]. Furthermore, bombardment of argon ions also generates stacking faults on $\{111\}$ plane of TiN crystals and stacking fault probability increases with increasing bias voltage (i.e. with increasing argon ion energy) beyond $-2 \mathrm{kV}$. Anisotropic diffraction line broadening in TiN thin films has already been observed by several researchers in the past [2-4]. In the present work, observed anisotropic line broadening may not be due to so called 'dislocation strain anisotropy' [13] because the 'modified W-H plot' [13] (constructed assuming both $\{111\}<110>$ and $\{111\}<211>$ dislocation slips separately in TiN thin films) indicates that FWHM $(\Delta K)$ is not monotonic function of $K C^{1 / 2}$ where $C$ is the average contrast factors of dislocations (for both screw and edge components) calculated using the single crystal elastic constants (anisotropic) of TiN along different diffraction vectors. Therefore, the observed line broadening anisotropy is perhaps due to direction dependent elastic grain interaction due to elastic anisotropy of TiN. The observed curvatures in $d$ $\operatorname{Sin}^{2} \psi$ plots also indicate such direction dependent grain interaction in textured TiN thin films. Evidence of anisotropic diffraction line broadening due to elastic grain interaction has recently been shown [14]. Bombardment of high energy argon ions leads to gradual alignment of [111] direction of TiN crystal with the substrate normal (Fig.3(c) \&(d)).

\section{Summary}

Effect of substrate bias voltage on the microstructure of TiN thin films has been studied by X-ray diffraction. Lattice parameter anisotropy $\left(a_{111}<a_{200}\right)$ are observed at all bias voltages. Lattice parameter shows the trend $a_{111}<a_{200}<a_{220}$ at higher bias voltages $(>-2 \mathrm{kV})$ due to preferential argon entrapment in TiN lattice. XRD line broadening analysis indicates strain anisotropy. $d-\operatorname{Sin}^{2} \psi$ plots shows pronounced curvature during stress measurement and TiN films are under tensile stresses ( $>-2 \mathrm{kV}$ bias voltage). Observed anisotropic line broadening is perhaps due to direction dependent elastic grain interaction in TiN thin films and not due to dislocation/dislocation loops (if any) in TiN films.

\section{References}

[1] J. E. Sundgren, Thin Solid Films 128(1985) 21

[2] R. Kuzel, Jr., R.Cerny, V.Valvoda, M.Blomberg and M.Merisalo, Thin Solid Films, 247(1994) 64-78.

[3] R. Kuzel, Jr., R.Cerny, V.Valvoda, M.Blomberg and M.Merisalo ,S. Kadlec, Thin Solid Films, 268(1995) 72-82.

[4] V. Valvoda, A.J.Perry, L.Hultman,J.Musil, S.Kadlec,Surface and coatings Technology, 49 (1991) 181-187

[5] Noyan I. C., Cohen J. B. Residual Stress, (Springer-Verlag New York Inc. 1987)

[6] R.P.I. Adler, H.M.Otte and C.N.J.Wagner, Metallurgical Transactions, 1 (1970) 2375-2382

[7] C. N. J. Wagner: Local atomic arrangements studied by X-ray diffraction, J.B. Cohen and J.E.Hilliard, Eds. P.219, Gordon and Breach, New York, 1966

[8] A. J. Perry, Thin Solid Films, 193 (1990) 463-471.

[9] G. K. Williamson and W. H. Hall, Acta Metall., 1 (1953) 22.

[10] U. Welzel, J. Ligot, P.Lamparter, A. C. Vermeulen, E. J. Mittemeijer J. Appl. Crystallogr. 38 (2005) 1.

[11] L. Hultman, G.Hakansson, U. Wahlstrom, J. E. Sundgren, I. Petrov, F. Adibi, J.E. Greene, Thin Solid Films, 205 (1991) 153-164

[12] I. Petrov, F. Adibi, J. E. Greene, L. Hultman and J. E. Sundgren Appl. Phys. Lett. 63(1) (1996) 36-38

[13] T. Ungar and A. Borbely, Appl. Phys. Lett. 69 (1996) 3173.

[14] M.K.A. Koker, U. Welzel and E. J. Mittemeijer, J. Appl. Cryst. 47 (2014) 391-401 